\title{
Length-scale-dependent stress relief mechanisms in indium at high homologous temperatures
}

\author{
Fereshteh Mallakpour ${ }^{1}$, Masoud Kasraie ${ }^{1,2}$, Erilk G. Herbert ${ }^{1, a)}$, P. Sudharshan Phani ${ }^{3}$, \\ Stephen A. Hackney ${ }^{1}$ \\ ${ }^{1}$ Department of Materials Science and Engineering, Michigan Technological University, Houghton, MI 49931, USA \\ ${ }^{2}$ Department of Mechanical Engineering, Michigan Technological University, Houghton, MI 49931, USA \\ ${ }^{3}$ International Advanced Research Centre for Powder Metallurgy and New Materials, Hyderabad, Telangana 500005, India \\ a) Address all correspondence to this author. e-mail: eherbert@mtu.edu \\ Received: 4 November 2020; accepted: 23 March 2021; published online: 14 April 2021
}

Erik G. Herbert and Stephen A. Hackney were editors of this journal during the review and decision stage. For the JMR policy on review and publication of manuscripts authored by editors, please refer to http://www.mrs.org/editor-manuscripts/

Nanoindentation and electron microscopy have been used to examine the length-scale-dependent stress relaxation mechanisms in well-annealed, high-purity indium at a homologous temperature of 0.69. The experimental methods, analysis, and observations serve as a stepping stone in identifying the stress relaxation mechanisms enabling the formation and growth of metallic dendrites originating at the buried interface between a metallic anode and a solid electrolyte separator. Indium's load-displacement data are found to be very similar to that of high-purity lithium. Residual hardness impressions show two distinct surface morphologies. Based on these morphologies, the measured hardness, and the estimated pile-up volume, it is proposed that residual impressions exhibiting significant pile-up are the result of deformation dominated by interface diffusion. Alternatively, impressions with no significant pile-up are taken to be the result of shear-driven dislocation glide. An analytical model is presented to rationalize the pile-up profile using interface diffusion.

\section{Introduction}

The use of nanoindentation to examine mechanical behavior at high homologous temperatures offers unique insights into creep processes at small length scales. Small length-scale creep is particularly important at non-planar interfaces between materials of different homologous temperatures, where a discontinuity in the bulk plastic relaxation mechanism can result in complex length-scale and strain rate-dependent mechanisms controlling the relaxation of stress gradients [1]. This has recently been demonstrated for the process of lithium metal nanoindentation at room temperature and related to the mechanical stability of the interface between a solid-state electrolyte (SSE) and the lithium metal negative electrode (anode) in solid-state batteries [1-3]. As previously reported, the self-limiting behavior of lithium at small length scales, where the probability of finding mobile dislocations or operable dislocation multiplication sources is low, is governed by a unique competition for stress relief between stress-directed diffusional flow and shear-driven dislocation glide. Within this poorly understood and minimally documented regime, the flow stress (hardness) of lithium is found to be strongly dependent on the strain rate and length scale (indentation depth) [2,3].

In this paper, we carry out a nanoindentation study of wellannealed, high-purity indium as a way to verify the previously reported lithium results using a more chemically stable surrogate, as indium has a standard electrode potential between $-0.34 \mathrm{~V}$ and $-0.49 \mathrm{~V}$ versus the standard hydrogen electrode (SHE) while lithium has a standard electrode potential of $-3.04 \mathrm{~V}$ versus SHE [4]. Moreover, the chemical stability of indium (relative to lithium) enables the utilization of additional imaging techniques outside the controlled atmosphere of an inert glove box. Table 1 directly compares the relevant physical, mechanical, and thermal properties of both indium and lithium [5]. While indium is clearly not a relevant anode material, the goal of this investigation is to document the experimental methods, observations, and 
TABLE 1: Constants \& properties of bulk indium and lithium and the interface diffusion model constants for indium.

\begin{tabular}{|c|c|c|}
\hline Constant/property \& model constants & Indium & Lithium \\
\hline Crystal structure & $\mathrm{BCT}$ & $\mathrm{BCC}$ \\
\hline$\rho\left(\mathrm{g} / \mathrm{cm}^{3}\right)$, density & 7.30 & 0.534 \\
\hline$E(G P a)$, elastic modulus ${ }^{1}$ & 12.7 & 9.5 \\
\hline$G(\mathrm{GPa})$, shear modulus & 3.8 & 4.25 \\
\hline$\nu(-)$, Poisson's ratio & 0.45 & 0.362 \\
\hline$\sigma_{y}(\mathrm{MPa})$, yield stress & 0.93 & 0.5 \\
\hline$T_{m}\left({ }^{\circ} \mathrm{C}\right)$, melting temperature & 156.6 & 180.5 \\
\hline$D_{o}\left(\mathrm{~m}^{2} / \mathrm{s}\right)$, pre-exponential term & $3.2 \times 10^{-4}$ & $2.3 \times 10^{-5}$ \\
\hline$Q_{d}(J)$, activation energy for self-diffusion & 78,300 & 55,300 \\
\hline$T\left({ }^{\circ} \mathrm{C}\right)$, test temperature & 24.4 & \\
\hline$T_{H}(-)$, homologous temperature at $24.4^{\circ} \mathrm{C}$ & 0.69 & 0.66 \\
\hline$D\left(\mathrm{~m}^{2} / \mathrm{s}\right)$, self-diffusion coefficient & $5.7 \times 10^{-18}$ & $4.5 \times 10^{-15}$ \\
\hline$D_{s}\left(\mathrm{~m}^{2} / \mathrm{s}\right)$, surface diffusivity & $5 \times 10^{-11}$ & NA \\
\hline$\delta(\mathrm{m})$, surface layer thickness & $1 \times 10^{-9}$ & NA \\
\hline$\Omega\left(\mathrm{m}^{3} /\right.$ atom $)$, atomic volume & $2.6 \times 10^{-29}$ & NA \\
\hline$\gamma\left(\mathrm{J} / \mathrm{m}^{2}\right)$, surface energy & 1 & NA \\
\hline$V(\mathrm{~m} / \mathrm{s})$, triple point velocity & $1 \times 10^{-8}$ & NA \\
\hline$H\left(\mathrm{~J} / \mathrm{m}^{3}\right)$, hardness & $5.4 \times 10^{7}$ & NA \\
\hline$\omega(1 / \mathrm{m})$, elastic relaxation length & $1.2 \times 10^{6}$ & NA \\
\hline$k_{B}(\mathrm{~J} /($ atom $\cdot \mathrm{K}))$ & $1.38 \times 10^{-23}$ & NA \\
\hline
\end{tabular}

${ }^{1}$ The elastic modulus of lithium is highly anisotropic.

rationalizations associated with the operation and identification of specific stress relaxation mechanisms in a high-purity, crystalline metal at a high homologous temperature and "small" length scales (indentation depths $\leq \sim 1 \mu \mathrm{m}$ ). In addition to its chemical stability, we note indium is particularly well suited for this purpose because it enables testing at high homologous temperatures without the complications of conducting high-temperature nanoindentation testing. In this way, the results reported here serve as a comparative stepping stone, enabling a more complete and comprehensive understanding of small length-scale creep, particularly as it relates to candidate anode materials, i.e., lithium and sodium, at length scales commensurate with the formation and growth of metallic filaments or dendrites.

In comparison to well-annealed elemental metals at low homologous temperatures, the load-displacement "pop-in" events observed in indium are entirely unique [6,7]. The pop-ins observed here and reported elsewhere do not represent the transition from elastic to elastic-plastic deformation, but rather an abrupt transition in the mechanism by which flow occurs in wellannealed, high-purity indium [6]. Collectively, the data presented here are generally consistent with the strain rate and length-scaledependent competition for stress relief previously reported in high-purity lithium. Prior to the pop-in event observed during nanoindentation, we submit the primary distinction between indium and lithium is the mechanism that controls flow, which

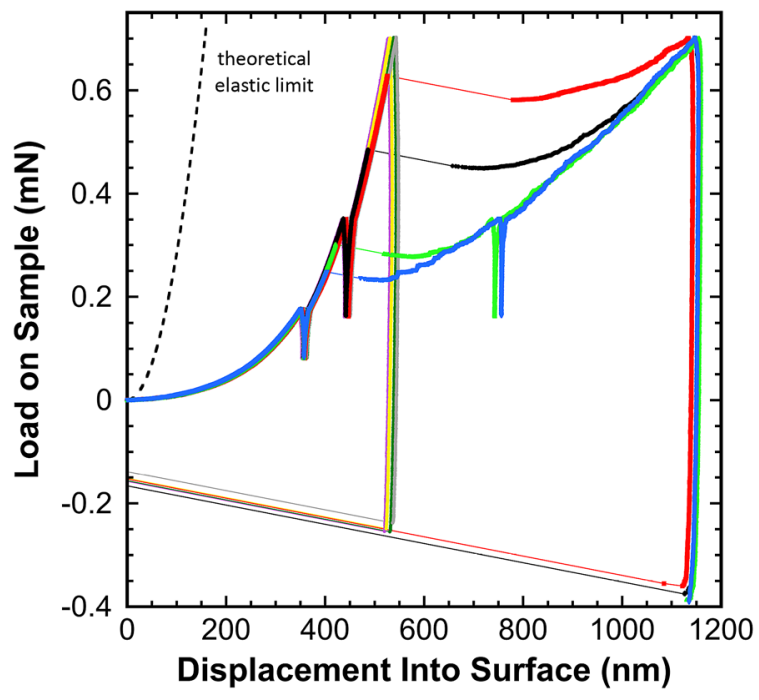

Figure 1: Representative cyclic load displacement curves in wellannealed, high-purity indium at a homologous temperature of 0.69 . The black dashed line represents the theoretical elastic load-displacement curve for the equivalent cone (centerline-to-face angle $=70.32^{\circ}$ ).

we propose is interface rather than volume diffusion, respectively $[8,9]$. While the results presented here provide unique insight into the stress-directed diffusional flow of indium, there are still many unanswered questions. Chief among them is a meaningful examination of strain rate effects in the limit of stress-directed diffusional flow, which awaits the evolution of constant strain rate testing capability under these unique deformation conditions.

\section{Experimental observations and discussion}

\section{Load-displacement curves}

Figure 1 shows 8 cyclic load-displacement, $P-h$, curves from a total of 25 measurements performed at a homologous temperature, $T_{H}$, of 0.69 . Among these representative curves, four exhibit a significant strain burst or "pop" and four do not. Considering the four curves that do pop, we note the stochastic nature of the event, as two curves pop between the first and second load-unload cycle, while the other two pop between the second cycle and final unload. Although not explicitly shown in Fig. 1, none of the $25 P-h$ curves exhibit a strain burst before completing the first load-unload cycle. Since the first cycle clearly exhibits elastic-plastic deformation (dominated by plasticity), we conclude the observed strain bursts are not representative of the onset of plastic deformation, but rather an abrupt, albeit stochastic transition in the stress relaxation mechanism by which indium accommodates plasticity under the imposed testing conditions. As a point of reference, the dashed black line originating at the 


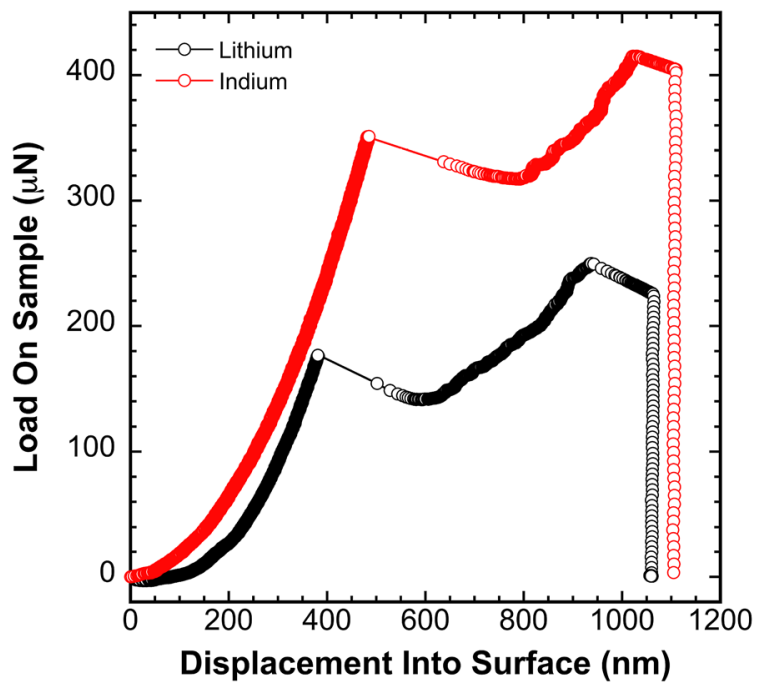

Figure 2: Representative load-displacement curves in well-annealed, high-purity indium and a high-purity, vapor-deposited $18 \mu \mathrm{m}$ thick lithium film.

origin illustrates the theoretical elastic $P-h$ curve for a sharp equivalent cone (half-included angle $=70.32^{\circ}$ ) $[1,2]$. As per Table 1, the theoretical curve assumes $E=12.7 \mathrm{GPa}$ and $v=$ 0.45 . As the plot shows, the elastic limit clearly indicates the measured $P-h$ curves are representative of elastic-plastic deformation.

For comparative purposes, Fig. 2 shows the unique similarities between representative $P-h$ curves for both indium and lithium (both near room temperature and subjected to similar strain rates). Specifically, we note the monotonically increasing load followed by the abrupt strain burst, serrated flow, extensive creep (see Ref. [2]), and the nearly vertical unload. These similarities suggest that near room temperature, high-purity indium and lithium accommodate plastic deformation by utilizing similar stress relaxation mechanisms.

Immediately prior to testing, the $\sim 3 \mathrm{~mm}$ thick indium specimen was annealed in an evacuated quartz cylinder at a homologous temperature, $T_{H}$, of 0.89 for $6 \mathrm{~h}$. Post-test, energy-dispersive $\mathrm{x}$-ray spectroscopy (EDS) was performed immediately after imaging the residual hardness impressions and revealed no significant oxygen or carbon contamination on the free surface. Through etch pit analysis, the resulting dislocation density at the surface, $\rho$, was determined to be $7.09 \times 10^{4} \mathrm{~cm}^{-2}$, which corresponds to an average dislocation spacing of $\rho^{-0.5}=37.6 \mu \mathrm{m}$. Figure 3 a shows representative etch pits in close proximity to the array of residual hardness impressions. In this well-annealed, low dislocation density condition, the $P-h$ curves are nominally expected to display a discernable strain burst or "pop-in" indicating the transition from elastic to elastic-plastic deformation [10]. Metallic specimens in a similar condition (well-annealed) frequently exhibit pop-ins at indentation depths ranging from $\sim 10 \mathrm{~nm}$ to
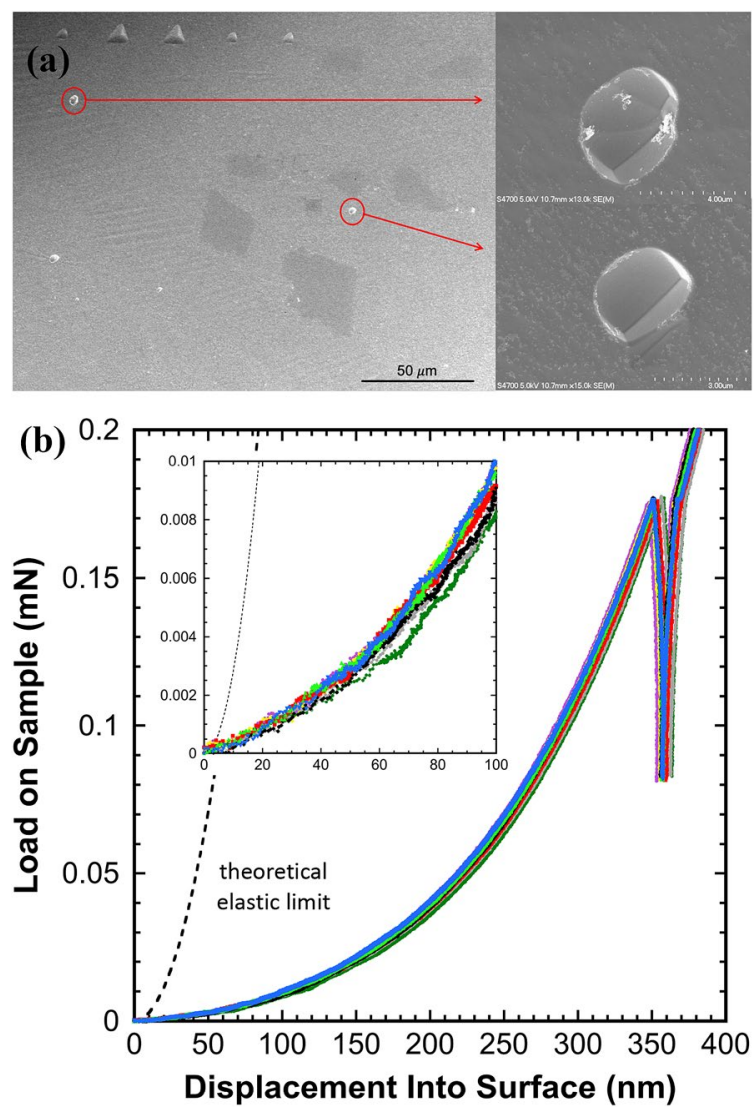

Figure 3: (a) Two representative etch pits and (b) eight representative load-displacement curves in indium, showing no evidence of the transition from elastic to elastic-plastic deformation (no pop-ins).

$200 \mathrm{~nm}$ [10]. The magnitude of the displacement burst often spans a similar range. Figure $3 \mathrm{~b}$, however, shows the $P-h$ curves are completely smooth and monotonically increasing to the first partial unload, giving no discernable evidence of the onset of plastic deformation. While an extremely sharp indenter tip could make the transition difficult to detect or perhaps even completely obscure it, the tip employed here is neither extremely sharp nor blunt. As shown in Fig. 4, the projected area, $A_{p}$, given by a mathematical description of the indenter tip's physical geometry (the area function) is found to match the $A_{p}$ of an infinitely sharp equivalent cone to within $10 \%$ or better only at contact depths $\geq \sim 200 \mathrm{~nm}$. Given the well-annealed condition of the test specimen, the sharp but not too sharp indenter tip geometry and the high $T_{H}$, the absence of pop-ins suggests the plastic deformation mechanism prior to the observed strain bursts may not be sheardriven dislocation glide, but rather a form of diffusive flow.

Details regarding the significant amount of additional load required to pull the indenter tip off the surface of the test specimen are not well understood. Among the potential experimental artifacts that could systematically contribute to the apparent adhesion between the indium surface and the diamond indenter 


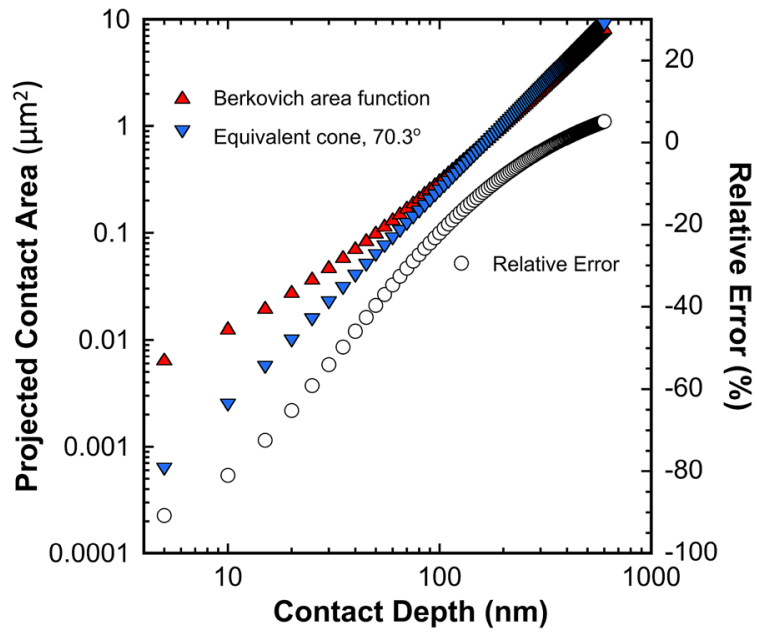

Figure 4: Direct comparison of the projected contact area between the Berkovich indenter tip's area function and an infinitely sharp equivalent cone.

tip, several can justifiably be eliminated: (1) Uncertainty in the surface detection is minimal, as the zero point is accurate to within $5 \mathrm{~nm}$ or better; (2) thermal drift is unlikely to be a significant source of error in the measured displacement because each individual experiment was performed in less than $60 \mathrm{~s}$ and the peak displacements are relatively large $(\geq 550 \mathrm{~nm})$; and (3) uncertainty in the frame stiffness is also an unlikely source of significant error in the measured displacement because the relatively large flat-backed test specimen is rigidly mounted to a glass slide with a thin layer of epoxy and the estimated elastic contact stiffness at the peak displacement of $1200 \mathrm{~nm}$ is $\sim 65 \mathrm{kN} / \mathrm{m}$ (assuming $E=12.7 \mathrm{GPa}$, the contact depth, $h_{c}=$ $1200 \mathrm{~nm}$, Poisson's ratio of indium, $v=0.45$, and the projected contact area, $A_{p}=13 \mu \mathrm{m}^{2}$ ), which is only $6.1 \%$ of the measured frame stiffness $\left(1.06 \times 10^{6} \mathrm{~N} / \mathrm{m}\right)$.

\section{Residual hardness impressions}

Images from a scanning electron microscope (SEM) in Fig. 5 show (a) the $5 \times 5$ array of residual hardness impressions in wellannealed, high-purity indium, (b) a representative residual hardness impression from one of the six measurements exhibiting a strain burst ( $45^{\circ} y$-axis tilt), (c) a representative impression from one of the 19 measurements that do not exhibit a strain burst $\left(45^{\circ}\right.$ $y$-axis tilt), and (d) a representative impression used to measure the projected contact area from a $P-h$ curve that did not exhibit a strain burst. The accuracy of the microscope's scale marker was experimentally verified using a single diffraction grating with $3 \mu \mathrm{m}$ spacing as well as the $30 \mu \mathrm{m} x-y$ spacing between the residual hardness impressions shown in Fig. 5a, which were placed by the instrument's piezo stages. In both cases, the measured distances were found to match the known values to within $3 \%$ or better.
Despite being subjected to the same maximum load and exhibiting indistinguishable $P-h$ curves prior to the strain burst, the SEM images and the corresponding $P-h$ data show the pop and no-pop curves correlate to significantly different contact dimensions and strikingly different surface morphologies. As shown in Fig. 5b, the $P-h$ curves exhibiting the pop create a larger contact and a surface morphology characterized by little to no discernable pile-up, straight, well-defined contact edges and distinct hemispherical slip traces in the free surface that appear as concentric rings radiating outward from 2 of the 3 faces of the residual hardness impression (plastic anisotropy). In contrast, Fig. $5 \mathrm{c}$ shows the no-pop $P-h$ curves create a significantly smaller contact and result in a surface morphology characterized by significant pile-up, curved contact edges and no discernable slip traces in the free surface. This direct comparison between the pop and no-pop residual hardness impressions and the corresponding $P-h$ curves clearly indicates a significant change in the stress relaxation mechanism operating before and after the strain burst. Furthermore, the characteristic features of the surface morphologies suggest the volume of material displaced by the indenter tip during the no-pop experiments may be uniquely conserved by the observed pile-up rather than the hinterland. In the analysis that follows, a new model is presented that rationalizes the observed pile-up based on interface diffusional flow.

In addition to the EDS analysis, we further note that images of the residual hardness impressions in Fig. 5 show no discernable evidence of cracking or spalling of an oxide layer. After annealing for $6 \mathrm{~h}$ at $T_{H}=0.89$, the diffusion length was approximately $12 \mu \mathrm{m}$ ( $D$ is given in Table 1 ). Although the average grain size was not formally measured, many of the grains were within the range of $2 \mathrm{~mm}$ to $3 \mathrm{~mm}$ in diameter. As such, the entire 5 $\times 5$ array of indents was performed near the center of a single grain, albeit with an unknown orientation. Assuming the important stress field beneath the indenter tip is constrained within a hemispherical radius that is 10 times the depth, then even at the maximum transition depth of $550 \mathrm{~nm}\left(10^{\star} 0.55 \mu \mathrm{m}=5.5 \mu \mathrm{m}\right)$, it is unlikely that prior to the indentation test, in its well-annealed condition, that the volume of indium to be sampled by the indenter encompassed a significant gradient in the dislocation density or residual stresses. Furthermore, if a uniform dislocation gradient did exist in the well-annealed condition, then presumably the $P-h$ data from indents within the same grain would generate a more repeatable pop-in depth from one test site to the next, not the stochastic response observed in Fig. 1.

\section{Hardness (the mean pressure the surface is capable of supporting)}

Figure 6a shows the measured hardness, $H,\left(H=P / A_{p}\right)$ of well-annealed, high-purity indium at a $T_{H}$ of 0.69 . In rationalizing this result, we consider the effect of pile-up, the depth 

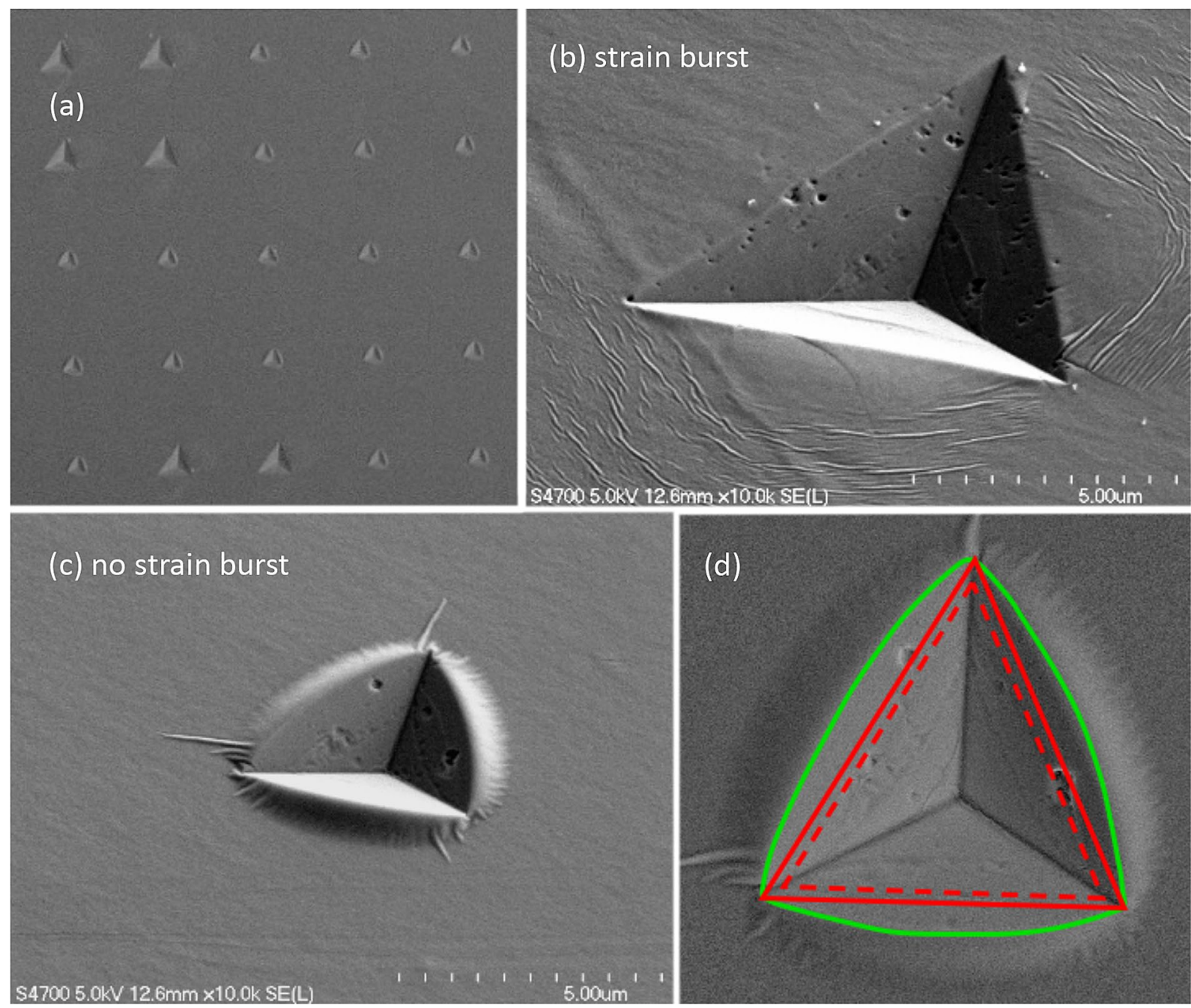

Figure 5: (a) The $5 \times 5$ array of residual hardness impressions. The indent spacing is $30 \mu \mathrm{m}$ in the $x$ and $y$ directions. (b and c), Representative impressions from indents that do and do not, respectively, exhibit a strain burst. The $y$-axis of the microscope stage is tilted by $45^{\circ}$ to enhance the contrast and visual perspective of the pile-up or lack thereof. (d) A representative illustration of the projected contact area. The dashed and solid red boundaries ignore pile-up and indicate the projected area based on the measured indentation depth and the residual impression, respectively. The solid green boundary shows the additional contact dimensions due to pile-up. The average side length (corner-to-corner) from the residual hardness impression (solid red line) is $4.6 \mu \mathrm{m} \pm 4.9 \%$.

dependence and the magnitude of the measured versus the nominally expected $H$. Capitalizing on indium's extremely high ratio of elastic modulus to yield strength, $\sim 13,700$ (Table 1), the $H$ data shown in Fig. 6a were calculated by intentionally ignoring the elastic deflection of the free surface and assuming that the contact depth, $h_{c}$, is accurately approximated by the indentation depth, $h$ [2]. In other words, the $H$ was calculated by assuming $h_{c}$ is in the original plane of the surface. Based on the images of the residual hardness impressions, this simplifying assumption is clearly only valid at the maximum load, $P_{\max }$, for the measurements that exhibit a strain burst, as they do not show any discernable evidence of pile-up or sink-in. For the measurements that do not exhibit a strain burst, an accurate estimate of $H$ at $P_{\max }$ can only be obtained by accounting for the pile-up through a direct a measurement of $A_{p}$ from the unloaded residual hardness impression. Fig. $5 \mathrm{~d}$ shows concentric outlines of three projected areas used in analyzing the $P-h$ curves that do not exhibit a strain burst. The outer most outline in green represents the $A_{p}$ adjusted for pile-up. Once the pile-up is considered, the $A_{p}$ at the indentation depth of $550 \mathrm{~nm}$ increases from $7.1 \mu \mathrm{m}^{2}$ to $13.0 \mu \mathrm{m}^{2}$. Correspondingly, the peak $H$ decreases by $48 \%$, from $\sim 103 \mathrm{MPa}$ to $\sim 54 \mathrm{MPa}$. At the maximum depth of $1150 \mathrm{~nm}$, the SEM image of the residual hardness impression, Fig. 5b, shows no discernable evidence of pile-up or sinkin (the pile-up is presumably consumed by the strain burst). As expected, the $A_{p}$ measured from the SEM image and the $A_{p}$ determined by assuming $h_{c}=h$ are found to match within 

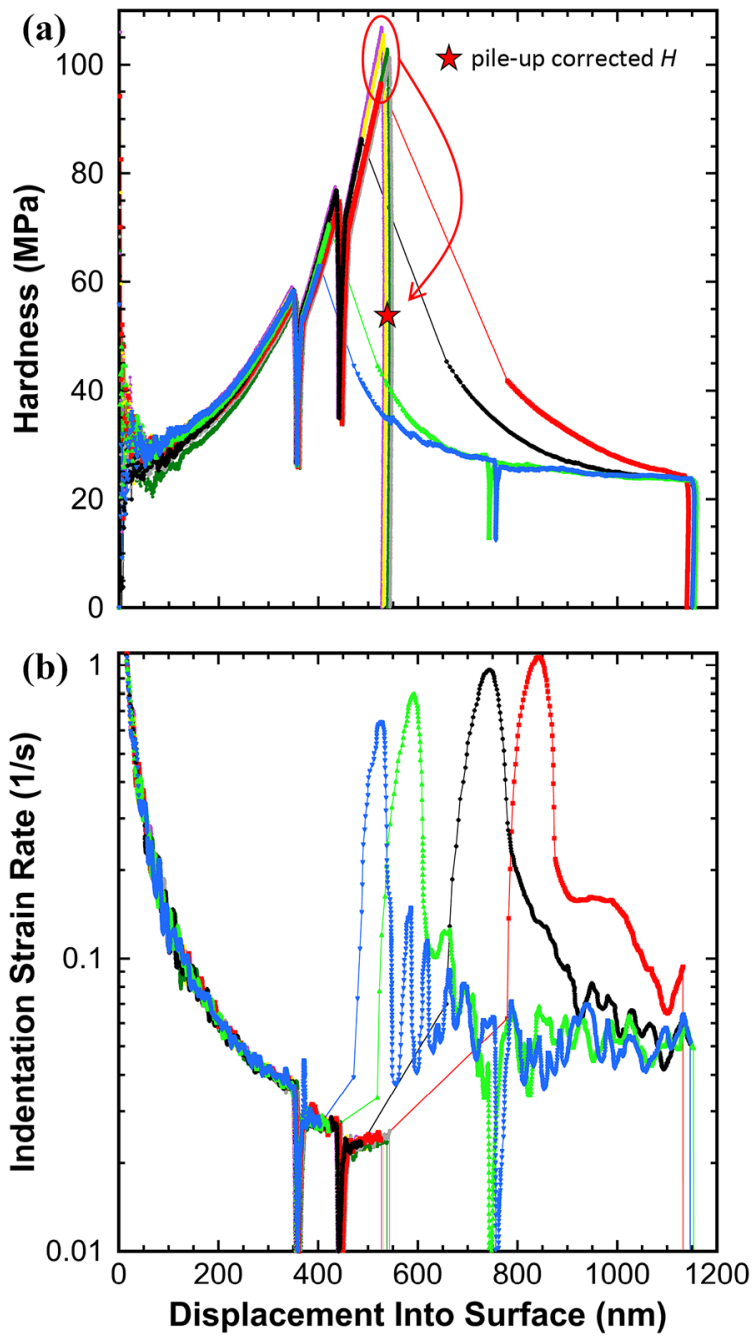

Figure 6: (a) The measured hardness as a function of depth and (b) the corresponding indentation strain rate. The red star in (a) represents the average hardness at the peak load based on the measured area from a residual hardness impression, which directly considers the increase in the projected contact area due to pile-up.

$10 \%$. The reported $H$ of $\sim 24 \mathrm{MPa}$ is, therefore, taken to be a reasonable estimate of the $H$ or the mean pressure the surface is capable of supporting at $h=1150 \mathrm{~nm}$.

At indentation depths large enough to avoid the welldocumented indentation size effect, ISE, $(h \geq \sim 1 \mu \mathrm{m}$ to $3 \mu \mathrm{m})$ the nominally expected $H$ of a bulk, polycrystalline metal is 3 times the metal's flow stress, $\sigma_{f}$, at the characteristic strain imposed by the indenter tip geometry (nominally $8 \%$ for the Berkovich) [10]. Given the high $T_{H}$ of 0.69 , the effect of work hardening is taken to be minimal and, thus, we assume $\sigma_{f}=\sigma_{y}$. As such, the nominally expected bulk $H=3 \sigma_{y}=3(0.93 \mathrm{MPa})$ $=2.8 \mathrm{MPa}$. In comparison, the pile-up corrected peak $H$ of $\sim$ $54 \mathrm{MPa}$ is nearly 20 times larger. Assuming the theoretical shear strength of indium lies within the range of $G /(2 \pi)$ to $G / 30$, where $G$ is the shear modulus of indium $(G=3.8 \mathrm{GPa})$, we note $54 \mathrm{MPa}$ is at most $43 \%$ of indium's theoretical shear strength.

While the peak $H$ of $\sim 54 \mathrm{MPa}$ occurs at a depth $(h=\sim$ $550 \mathrm{~nm}$ ) well within the expected intrinsic ISE window, we reject the ISE as a potential explanation for the difference between the measured and expected $H$. As previously noted, the high $T_{H}$, the low dislocation density, the lack of pop-ins in the $P-h$ curves, and the apparent frustration of dislocation-mediated flow collectively suggest a dominant flow mechanism other than dislocation glide, which is a fundamental tenet of the ISE [10]. Nevertheless, neglecting these observations and assuming the deformation prior to the strain burst is mediated by dislocation glide, then an intrinsic ISE would require a breakdown in geometric self-similarity of the contact such that the observed depth dependence prior to the strain burst is completely reversed. While not impossible, this scenario seems highly improbable.

An alternative explanation for the observed discrepancy between the measured and expected $H$ is that plastic deformation prior to the observed strain burst is mediated by a less efficient flow mechanism such as stress-directed diffusion rather than dislocation glide. In this context, the comparison between the measured $H$ and $3 \sigma_{y}$ is invalid. While models capable of predicting the measured $H$ in terms of diffusional flow do exist, they are currently limited to volume diffusion [1-3]. As will be examined in a subsequent section, here we propose the dominant plastic deformation mechanism is interface rather than volume diffusion (both have a stress exponent, $n$, of 1 ) $[2,7,8]$. In the absence of a model that incorporates this mechanism and enables quantitative comparisons, here we qualitatively note that in comparison to dislocation glide, diffusive flow naturally depends on the diffusion length and, therefore, becomes an increasingly less efficient stress relaxation mechanism as the diffusion length increases with indentation depth [2, 6, 7]. We also note that within the limit of diffusional flow, it is possible that geometric self-similarity of the contact is not lost. Although clearly speculative, this outcome would potentially provide a simple means of accounting for pile-up and correcting the nominally measured $H$ as a continuous function of depth. In this way, the measured $H$ at $h \leq 550 \mathrm{~nm}$ would be uniformly decreased by $48 \%$, noting, however, that potential strain rate effects have not been considered. While elucidating rate effects is clearly a necessary and critical step forward, it is important to understand that conventional constant strain rate nanoindentation loading algorithms, such as the $\dot{P} / P$ technique used here, by definition, cannot generate a constant strain rate at any length scale wherein the hardness is depth dependent. To that end, unambiguous examination of the competing effects of length scale and strain rate await the development of novel testing methods that are capable of controlling and maintaining a constant indentation strain rate under the unique stress-directed diffusional flow conditions observed here. 
As previously reported, the $H$ of $\sim 24 \mathrm{MPa}$ at the maximum depth of $1150 \mathrm{~nm}$ is taken to be an accurate estimate of the mean pressure the surface is capable of supporting. Although the deformation at this depth is presumably dominated by dislocation glide, we note the measured $H$ is still 8.6 times larger than the nominally expected $3 \sigma_{y}=2.8 \mathrm{MPa}$ (a constraint factor of nearly 26 rather than the expected value of 3 ). Among the factors potentially contributing to this discrepancy are an intrinsic ISE and strain rate sensitivity. Following an idea first proposed by Rester et al., it is also possible that following the transition or pop-in, dynamic recrystallization enables geometrically necessary dislocations (brought about by the gradient in strain directly beneath the indenter tip) to coalesce into cell walls or subgrains, thus providing an additional strengthening mechanism [11]. Although the $T_{H}$ of 0.69 is well within the hot working regime, it is also plausible that our assumption of negligible work hardening immediately following the burst is inaccurate. Indium's self-diffusion coefficient is quite small and, therefore, it is not clear how efficient or inefficient the recovery process is over the time scale from the strain burst to the maximum load ( $\leq 20 \mathrm{~s}$ ). The potential effect of strain rate is difficult to quantify, as indium's strain rate sensitivity at these length scales and under diffusional flow is entirely unknown [12, 13]. For the sake of posterity, Fig. $6 \mathrm{~b}$ shows the depth-dependent indentation strain rate, defined as the time rate of change of the displacement divided by the displacement, $h / h$. Over the indentation depths examined here, Fig. $6 \mathrm{~b}$ shows the strain rate spans nearly two orders of magnitude, from $\sim 1 \mathrm{~s}^{-1}$ to $0.02 \mathrm{~s}^{-1}$. We note the peak strain rate following the burst is an underestimation, as the calculated velocity utilizes an effective time constant of $\sim 0.2 \mathrm{~s}$.

Due to the small number of measurements (25 test sites), rigorous analysis of the stochastic nature of the strain burst is beyond the scope of this investigation. However, based on the striking similarities between the $P-h$ behavior of indium and lithium shown in Fig. 1b, here we adopt the same rationalization of the strain burst. Specifically, the strain burst is taken to represent an abrupt transition from diffusion to dislocation-mediated flow. Given the stochastic nature of the transition and the low dislocation density, we speculate that the sudden event is triggered by the combination of a critical threshold in stress and a sufficient length scale, as the dislocation mechanism clearly appears to require not only the necessary driving force, but sufficient room to physically operate [2].

\section{Volume versus interface diffusion}

Enabled by a high $T_{H}$ and a low dislocation density, we submit that diffusive flow is the most efficient stress relaxation mechanism at length scales that are small in relation to the spacing between mobile dislocations and/or operable dislocation multiplication sources. As presented in Table 1, indium's self-diffusion coefficient, $D$, at the test temperature of $24.4^{\circ} \mathrm{C}$ is $5.7 \times 10^{-18}$ $\mathrm{m}^{2} / \mathrm{s}$. This coefficient is quite small and suggests that volume diffusion to the free surface may not be an efficient transport mechanism, particularly as the diffusion length increases with indentation depth. Interface diffusion, on the other hand, requires approximately $1 / 2$ to $2 / 3$ the activation energy of volume diffusion. Assuming $Q_{\text {dinterface }}=Q_{\text {dvolume }} / 2$, then the corresponding interface diffusion coefficient is $4.29 \times 10^{-11} \mathrm{~m}^{2} / \mathrm{s}$. Here we assume the interface diffusion layer is $0.5 \mathrm{~nm}$ thick and that the relevant interface volume is the product of the thickness and the interface surface area under pressure. At an indentation depth of $550 \mathrm{~nm}$, previous analysis indicates the interface volume fraction is $6.62 \times$ $10^{-5}$ [2]. The effective diffusion coefficient given by Tilley is

$$
D_{\text {effective }}=(1-f) D_{\text {volume }}+f D_{\text {interface }}
$$

and, thus, $D_{\text {effective }}=2.8 \times 10^{-15} \mathrm{~m}^{2} / \mathrm{s}$, which is three orders of magnitude larger than volume diffusion alone [14]. Alternatively, if the ratio is only $2 / 3, D_{\text {effective }}$ is still more than an order of magnitude larger. This simple analysis indicates the diffusive flow mechanism for indium at $24.4^{\circ} \mathrm{C}$ is dominated by interface rather than volume diffusion. For the sake of comparison, we note the opposite is true of lithium at a similar temperature. Even at a substantially smaller depth, $150 \mathrm{~nm}$, the interface term is found to contribute only $4 \%$ to the effective diffusion coefficient. As indicated in Table 1, the apparent explanation for this difference is lithium's self-diffusion coefficient, which is nearly three orders of magnitude larger than that of indium.

\section{Quantifying the observed pile-up}

To create a two-dimensional profile of the pile-up, a line scan performed in the SEM was used to deposit a thin line of carbon through a residual hardness impression. By aligning the carbon line with the $y$-axis of the SEM stages and then tilting the $y$-axis by $45^{\circ}$, direct measurements of the $x-y$ coordinates of the carbon line were used to estimate the pile-up profile. While a properly calibrated atomic force microscope could presumably simplify this task, one was not readily available. Figure 7 shows the line scan oriented along the $y$-axis and tilted by $45^{\circ}$. The measured pile-up profile is shown in Fig. $7 \mathrm{~b}$.

Based on the recorded $P-h$ data and measurements from the SEM images of the in-plane dimensions of the residual hardness impressions and the pile-up profile, Fig. 8a shows a two-dimensional rendering of the contact geometry relative to the original plane of the surface. To capture key features of the geometry, the image presents an overlay that combines elements from three different cross-sections through (1) the center of an indent, (2) along an edge (corner-to-corner), and (3) along the ridge of the pile-up (corner-to-corner). The color coding corresponds to the residual hardness impression shown in Fig. 5d. In an attempt to compensate for the 


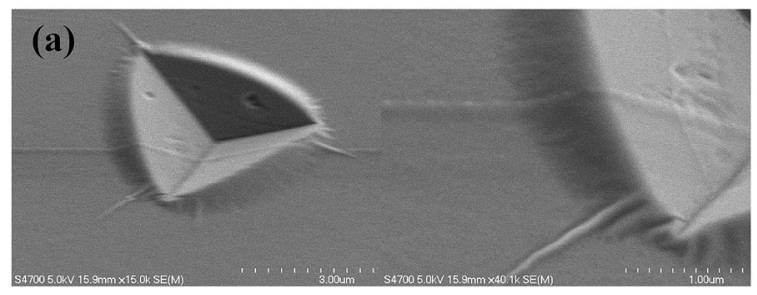

(b)

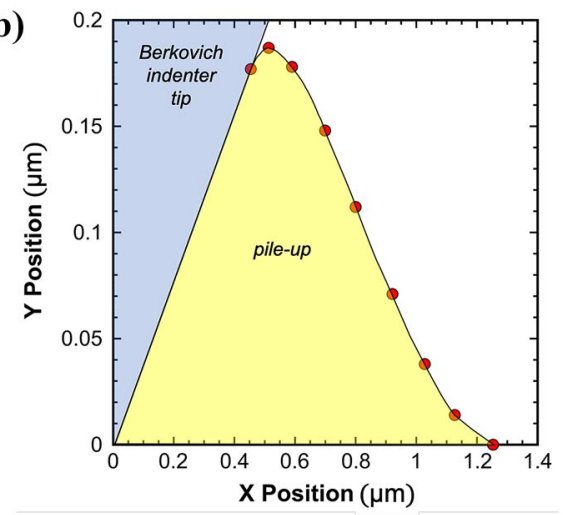

Figure 7: (a) The deposited carbon (line scan) through a residual hardness impression. The $y$-axis of the microscope stage is aligned parallel with the carbon line and tilted by $45^{\circ}$. (b) The measured pile-up profile as determined by measuring the $x-y$ coordinates of the carbon line.

(a)

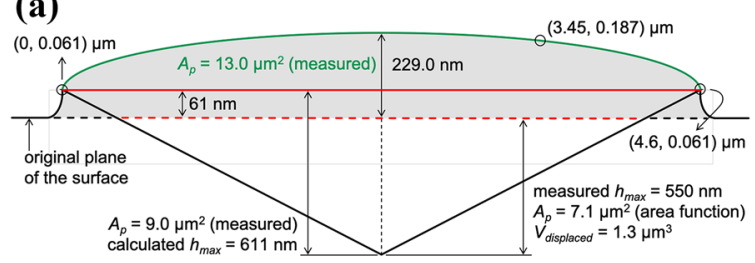

(b)

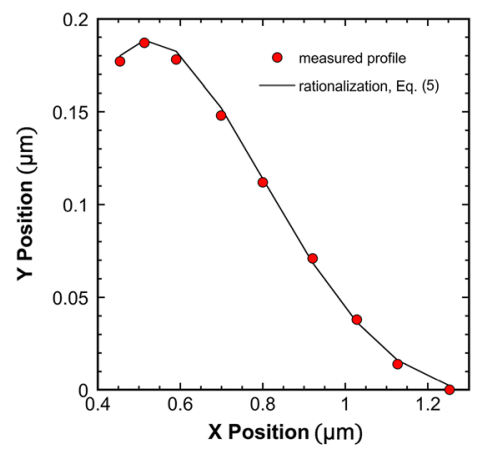

Figure 8: (a) A two-dimensional rendering of the contact geometry based on an overlay of three cross-sections through (1) the center of an indent, (2) along an edge (corner-to-corner), and (3) along the peak of the pile-up (corner-to-corner). (b) Rationalization of the pile-up profile using Eq. (5), which is based on interface diffusion.

off-center line scan, the peak pile-up height at the center of the face was estimated by fitting a parabola to the three points indicated along the ridge of the pile-up. As measured in the SEM, the average side length (corner-to-corner) and the position of the carbon line from the furthest corner were found to be $4.6 \mu \mathrm{m} \pm 4.9 \%$ and $3.45 \mu \mathrm{m}$, respectively. As per the parabolic fit, the peak pile-up height at the center of the face is estimated to be $229.0 \mathrm{~nm}$, which is $\sim 22.5 \%$ larger than the profile height measured from the line scan. Among the interesting observations, we note the $A_{p}$ calculated by the indenter tip's area function at $h=550 \mathrm{~nm}$ underestimates the measured $A_{p}$ (assuming no pile-up) by $21 \%$. Based on the elevated corners (relative to the original plane of the surface) of the residual impressions shown in Figs. $5 \mathrm{c}$ and $7 \mathrm{a}$, we propose this outcome is due to the flow of indium along the faces and below the corners of the indenter tip. In this way, the complete triangular residual hardness impression can physically climb above the original plane of the surface as depicted in Fig. $8 \mathrm{a}$. In other words, we submit the pile-up is not limited to occurring only along the faces of the indenter tip, as one might intuitively expect.

\section{A simple look at conservation of volume}

If the proposed stress relaxation mechanism is interface diffusion, then the volume of indium displaced by the indenter tip must be locally conserved by the pile-up. Limited by a single profile of the pile-up, our analysis is constrained to a rudimentary examination of the pile-up volume. As indicated by Fig. $8 \mathrm{a}$, the volume of indium displaced by the indenter tip relative to the original plane of the surface is $1.3 \mu^{3}$. As described above, here we assume the ridge of the pile-up is reasonably approximated by the parabola $y=0.061+0.14609 x-0.031758 x^{2}$, where $y$ and $x$ are in units of $\mu \mathrm{m}$ and physically represent the height of the pile-up relative to the original plane of the surface and the horizontal position along the face, respectively. At the position of the line scan $(x=3.45 \mu \mathrm{m}$ as measured in the SEM), the radius of a circle whose area is equivalent to the area under the profile is found to be $0.196 \mu \mathrm{m}$. Thus, the ratio of the pile-up height to equivalent radius is 0.955 . Assuming that ratio is constant over the side length of the residual hardness impression, then the volume of the pile-up along all three faces is given by the definite integral,

$$
V_{\text {pile-up }}=3 \pi \int_{0}^{4.6}\left(0.063893+0.15302 x-0.033264 x^{2}\right)^{2} d x,
$$

where the limits correspond to the average corner-to-corner side length and the integrand gives the equivalent radius as a function of position $(x)$ along each face. In this way, the estimated volume of the pile-up is found to be $1.54 \mu \mathrm{m}^{3}$, which compares reasonably well with the displaced volume of $1.3 \mu \mathrm{m}^{3}$ (the relative error is $18.5 \%$ ). This analysis demonstrates the distinct possibility that the displaced volume is uniquely conserved by the pile-up. 


\section{Rationalizing the pile-up via interface diffusion}

Interface diffusion along the faces of the indenter tip will be driven by gradients in the chemical potential at the free surface of the indium and at the interface between the indenter and the indium. For diffusion at the free surface, it is considered that the chemical potential, $\mu(\mathrm{J} / \mathrm{kg})$, is a function of both the surface strain energy, $\sigma^{2} / 2 E$, and the surface curvature, $\kappa(1 / \mathrm{m})$, where $\sigma$ is the stress at the free surface (normal components are null) and $E$ is the elastic modulus [15]. This may be written as

$$
\mu=\mu_{o}+\frac{\sigma^{2} \Omega}{2 E}-\Omega \gamma \kappa
$$

where $\Omega\left(\mathrm{m}^{3} /\right.$ atom $)$ is the atomic volume and $\gamma\left(\mathrm{J} / \mathrm{m}^{2}\right)$ is the surface energy.

Here, we use a quasi-steady state solution to describe the surface profile motivated by Mullins, where the surface profile depends on a constant (or slowly changing) triple point velocity, $V$ [16]. The original treatment by Mullins described a quasisteady-state profile for a moving grain boundary-surface triple junction, which is modified here to apply to the triple junction of the indenter-indium interface, indium surface, and vapor phase with inclusion of the elastic strain energy term. From this approach, the surface profile, $y(x)$, is obtained from the solution of the differential equation

$$
0=\frac{D_{s} \delta \Omega}{k_{B} T}\left(\frac{H^{2}}{E} \omega^{2} \exp (-\omega x)-\gamma \frac{d^{4} y}{d x^{4}}\right)+V \frac{d y}{d x},
$$

where $x$ is the horizontal surface position in a moving coordinate system of velocity, $V$, which is the triple point velocity with the indenter/indium triple point at the origin, $D_{s}\left(\mathrm{~m}^{2} / \mathrm{s}\right)$ is the surface diffusivity, $\delta$ is the surface layer thickness, $H$ is the hardness, $T$ is the temperature, $k_{B}$ is the Boltzmann constant, and $1 / \omega$ is a relaxation length for the elastic stress. A general solution to Eq. (4) is of the form

$$
\begin{aligned}
y= & C \exp (-\omega x)+A \exp \left(-\frac{\lambda x}{2}\right) \cos \left(\frac{\sqrt{3}}{2} \lambda x\right) \\
& +B \exp \left(-\frac{\lambda x}{2}\right) \sin \left(\frac{\sqrt{3}}{2} \lambda x\right) .
\end{aligned}
$$

The substitution of Eq. (5) into Eq. (4) defines the coefficients $C$ and $\lambda$ in terms of physical parameters. Grouping the parameters into $a$ and $b$ gives

$$
\begin{gathered}
a(-)=\frac{H^{2} \omega^{2}}{E} \frac{D_{s} \delta \Omega}{k_{B} T V} \\
b\left(\mathrm{~m}^{3}\right)=\frac{\gamma D_{s} \delta \Omega}{k_{B} T V} .
\end{gathered}
$$

TABLE 2: Comparison of the physical estimates and fitting parameters.

\begin{tabular}{lll}
\hline \hline Parameter & Physical estimate & Fitting parameter \\
\hline$a(-)$ & 0.01 & $\mathrm{NA}$ \\
$b\left(\mathrm{~m}^{3}\right)$ & $3.17 \times 10^{-20}$ & $\mathrm{NA}$ \\
$C(\mathrm{~m})$ & $8.27 \times 10^{-9}$ & $6.0 \times 10^{-8}$ \\
$\lambda(1 / \mathrm{m})$ & $3.16 \times 10^{6}$ & $4.7 \times 10^{6}$ \\
$\omega(1 / \mathrm{m})$ & $1.2 \times 10^{6}$ & $1.2 \times 10^{6}$ \\
$A(\mathrm{~m})$ & $\mathrm{NA}$ & $1.2 \times 10^{-7}$ \\
$B(\mathrm{~m})$ & $\mathrm{NA}$ & $1.5 \times 10^{-7}$ \\
\hline \hline
\end{tabular}

The parameters of $C$ and $\lambda$ can be then determined as

$$
\begin{gathered}
C(\mathrm{~m})=\frac{a}{b \omega^{4}+\omega} \\
\lambda\left(\mathrm{m}^{-1}\right)=\left(\frac{1}{b}\right)^{\frac{1}{3}},
\end{gathered}
$$

where $\omega$ is the inverse of the indentation depth (based on the Saint-Venant principle). Using the carbon line profile presented in Fig. 7b, Eq. (5) can be fit to the indium pile-up profile as shown in Fig. 8b. Admittedly, there are a large number of fitting parameters available to produce the close fit shown in Fig. 8b, but as discussed above, Eqs. (6-9) provide a physical basis for three $(\omega, C$, and $\lambda)$ of the five fitting parameters. A comparison of the fitting parameter values against the estimated values is shown in Tables 1 and 2, where the parameters are found to be within an order of magnitude with respect to the physical estimates.

In future studies, the proposed rationalization provides a unique opportunity to examine geometric self-similarity as well as the functional relationship between the measured properties, the contact dimensions, and the mechanism(s) of plastic deformation. Of particular interest are the effects of temperature, strain rate, and indenter tip geometry. Collectively, the rationalization proposed here and the existing $P-h$ relationships based on volume diffusion provide an extensive framework capable of providing new insight into small lengthscale creep mechanisms and the conditions that inhibit and/ or promote the formation and growth of metallic filaments or dendrites originating at the interface between a metallic anode and a solid electrolyte separator.

\section{Summary and conclusions}

Nanoindentation in well-annealed, high-purity indium was performed under the following conditions: Berkovich indenter tip, cyclic loading that was terminated at $175 \mu \mathrm{N}, 350 \mu \mathrm{N}$, and 
$700 \mu \mathrm{N}$, load control such that the loading rate divided by the load was targeted at $0.1 \mathrm{~s}^{-1}$, measured indentation strain rates varying from $0.02 \mathrm{~s}^{-1}$ to $1 \mathrm{~s}^{-1}$, and a homologous temperature of $0.69\left(24.4^{\circ} \mathrm{C}\right)$.

(1) The load-displacement curves and corresponding residual hardness impressions show the plastic deformation mechanism varies significantly with indentation depth and test site. Among 25 indents, 19 (76\%) reach the peak load of $700 \mu \mathrm{N}$ with no discontinuity in the measured load-displacement curve. The corresponding residual hardness impressions are characterized by bowed contact edges, significant pile-up, and no discernable slip traces around the periphery of the contact. Despite being terminated at the same maximum load, the remaining 6 indents (24\%) experience a stochastic strain burst and form a residual hardness impression that is approximately twice as deep, characterized by well-defined, straight contact edges, no discernable pile-up and slip traces in the free surface around 2 of the 3 indenter faces. Based on these observations and the estimated dislocation spacing of $37.6 \mu \mathrm{m}$, we submit the strain burst represents an abrupt transition from interface diffusion to dislocation-mediated flow. Given the stochastic nature of the transition and the low dislocation density, we speculate that the sudden event is triggered by the combination of a critical threshold in stress and a sufficient length scale, as the dislocation mechanism clearly appears to require not only the necessary driving force, but sufficient room to physically operate.

(2) At the peak load of $700 \mu \mathrm{N}, 19$ of 25 targeted test sites (76\%) did not experience a strain burst, terminated at a depth of $\sim 550 \mathrm{~nm}$ and yielded a measured hardness (pile-up corrected) of $\sim 54 \mathrm{MPa}$. The remaining 6 test sites (24\%) did experience a strain burst, terminated at a depth of $\sim 1150 \mathrm{~nm}$ and yielded a measured hardness of $\sim 24 \mathrm{MPa}$ (no pile-up or sink-in correction was deemed necessary). Based on the substantially higher hardness and extensive pile-up indicative of the test sites that do not experience a strain burst, we propose the dominant stress relaxation mechanism prior to the strain burst is interface diffusion rather than dislocation-mediated glide. Interface rather than volume diffusion is favored because the effective interface diffusion coefficient is 1 to 3 orders of magnitude larger than volume diffusion alone. Although the analysis is rudimentary, the estimated pile-up volume is found to be within $18.5 \%$ of the volume of indium displaced by the indenter tip. This suggests the plastically deformed volume of material may be uniquely conserved by the pile-up.
(3) The measured pile-up profile is rationalized using a new analytical model based on interface diffusion. The model is derived from Mullins' analysis of a moving grain boundary. Among the model's 5 fitting parameters, 3 are defined in terms of material or experimental parameters. This model, in conjunction with previously derived volume diffusion load-displacement relationships, expands the analytical framework to examine small length- scale creep mechanisms. This capability will serve to develop new insight into the stress relaxation mechanisms operating at the interface between a metallic anode and a solid electrolyte separator.

\section{Experimental methods}

\section{Preparation of well-annealed, high-purity indium}

The nanoindentation test specimen $(\sim 20 \times 15 \times 3 \mathrm{~mm})$ was cast from $99.999 \%$ pure indium shot (Indium Corporation, Clinton, New York). Approximately $15 \mathrm{~mm}, 3 \mathrm{~mm}$ pellets were melted in a quartz crucible on a hot plate at $\sim 200^{\circ} \mathrm{C}$. To eliminate adhesion between the cast indium and substrate, the molten indium was poured directly onto a room temperature aluminum block (diameter $=25.4 \mathrm{~cm}$, thickness $=7.6 \mathrm{~cm}$ ) covered with Kapton tape $\sim 1 \mathrm{~mm}$ thick. Within minutes of casting, the indium specimen was enclosed in an evacuated quartz tube and annealed for $6 \mathrm{~h}$ at $110^{\circ} \mathrm{C}$ (a homologous temperature of 0.89). At short wavelengths, the annealed, as-cast surface finish enabled reproducible load-displacement curves at indentation depths as small as $10 \mathrm{~nm}$. Using a thin layer of two-part epoxy resin, the annealed, as-cast indium specimen was mounted onto glass slides $(2 \times 2 \mathrm{~cm})$ previously crystal bonded (thermal set wax) to the instrument's 1.25 -inch aluminum specimen mounts.

In the interest of informing future experimentalists, we note that longer wavelength undulations in the as-cast surface made it practically impossible to successfully run multiple arrays within a single batch of experiments. A potential solution to this problem was recently presented by Fincher et al., who utilized lubricated glass slides and a benchtop vice to create parallel top and bottom surfaces of a sodium test specimen [17]. Although implementing this approach with indium will require extensive annealing, this path is particularly attractive in that it potentially provides a means to achieving a large test surface that is both smooth and flat.

\section{Nanoindentation}

Room temperature nanoindentation experiments were performed using a Berkovich diamond indenter tip mounted in the InForce50 nanomechanical actuator (Nanomechanics, Inc.KLA Tencor, Oak Ridge, Tennessee). Twenty-five measurements were targeted in a $5 \times 5$ array with $30 \mu \mathrm{m}$ spacing in the $x$ and $y$ 
directions. The maximum load was prescribed at $700 \mu \mathrm{N}$ and the loading was controlled such that the ratio of $\dot{P} / P$ was targeted at the user defined value of $0.1 \mathrm{~s}^{-1}$. The loading was performed in 3 cycles. The first two unloaded partially (50\%) from $175 \mu \mathrm{N}$ and $350 \mu \mathrm{N}$ while the final cycle unloaded completely from $700 \mu \mathrm{N}$. Given the high homologous temperature and the specimen's propensity to creep, no attempt was made to measure the thermal drift rate or correct the measured displacement for thermal drift. To help mitigate the effect of drift, the environment's thermal stability was maximized by utilizing an 8 -h start delay and running the experiments overnight. Furthermore, the selected load-time history allowed each individual test to be executed in less than $60 \mathrm{~s}$.

\section{Scanning electron microscopy}

Images of the residual hardness impressions and etch pits were obtained using a field emission high-resolution scanning electron microscope, the Hitachi S-4700 FE-SEM (Schaumburg, IL, USA). This microscope was also used to perform the energydispersive X-ray spectroscopy and deposit the carbon line scan. With the carbon line oriented parallel to the $y$-axis of the SEM stage, the vertical height of the pile-up profile, $z$, was calculated as a function of position along the $y$-axis using the relation $z=\Delta x / \sin \theta$, where $\Delta x$ is the directly measured change in position along the $x$-axis and $\theta$ is the $y$-axis tilt, which was $45^{\circ}$. For the pile-up dimensions examined here, this technique provides a simple, quantitative means of determining the pile-up height without requiring built-in tilt corrections from the microscope.

\section{Etch pit analysis}

Following nanoindentation, the indium specimen was iteratively etched in 3, 4-min intervals using a $10 \%$ nitric acid solution. The etch pit density (EPD) was measured from an area approximately $0.03 \mathrm{~mm}^{2}$ located directly below the $5 \times 5$ array of residual hardness impressions. Following each iteration, the EPD was measured using an Olympus BX51M (Center Valley, PA, USA) optical microscope and the image analysis software, ImageJ. The Hitachi S-4700 FE-SEM was used to examine the morphology and faceting of several etch pits. Following iterations 2 and 3, the EPD decreased, as several pits disappeared, others combined and several grew. Thus, the most accurate estimate of the EPD, $7.09 \times$ $10^{4} \mathrm{~cm}^{-2}$, was determined from the first 4 -min interval. The subsequent decrease in the EPD with time is taken to be the result of over etching. As shown in Fig. 3a, it is interesting to note that there are no discernable pits in close proximity to the residual hardness impressions - neither the ones that experienced a strain burst, nor the ones that did not. One potential explanation is that dislocations created by the strain burst may have annealed out. Due to the
COVID-19 pandemic, the EPD was not measured until 6 months after the indentation experiments were performed. Given the high $T_{H}$ of 0.69 , it is possible that over 6 months, the recovery process and a diffusion length of $\sim 9.5 \mu \mathrm{m}$ may have eliminated any dislocations created by the strain burst. Following the previously presented argument made by Rester et al., it is also possible that following the transition or pop-in, an intrinsic ISE does play a role in that GNDs caused by the indentation geometry have coalesced into cell walls or subgrains [11]. Presumably, the subgrains would develop directly beneath the indenter tip, as the GNDs would be limited to operating within the local gradient in strain. In this way, the GNDs would not be visible at the free surface and, thus, not discernable by etch pit analysis techniques.

\section{Acknowledgments}

This research was sponsored jointly by the US Department of Energy, Office of Energy Efficiency and Renewable Energy's Advanced Battery Materials Research program (managed by Tien Duong), and by TARDEC, the US Army Tank Automotive Research Development and Engineering Center. EGH and PSP were also partially supported by the Indo-US Science \& Technology Forum (IUSSTF) through the Virtual Networked Center award JC-045/2018. EGH is also particularly grateful for start-up funding from the Department of Materials Science and Engineering at Michigan Technological University.

\section{Author contributions}

All authors contributed to the study conception and design. Material preparation, data collection, and analysis were performed by FM, MK, EGH, PSP, and SAH. The first draft of the manuscript was written by FM. All authors read and approved the final manuscript.

\section{Funding}

This research was sponsored jointly by the US Department of Energy, Office of Energy Efficiency and Renewable Energy's Advanced Battery Materials Research program (managed by Tien Duong), and by TARDEC, the U.S. Army Tank Automotive Research Development and Engineering Center. EGH and PSP were also partially supported by the Indo-U.S. Science \& Technology Forum (IUSSTF) through the Virtual Networked Center award JC-045/2018. EGH is also particularly grateful for start-up funding from the Department of Materials Science and Engineering at Michigan Technological University.

\section{Data availability}

The raw data files are available from the corresponding author upon request. 


\section{Declarations}

Conflict of interest The authors have no conflicts of interest to declare that are relevant to the content of this article.

\section{References}

1. E.G. Herbert, N.J. Dudney, M. Rochow, V. Thole, S.A. Hackney, On the mechanisms of stress relaxation and intensification at the lithium/solid-state electrolyte interface. J. Mater. Res. 34, 3593-3616 (2019)

2. E.G. Herbert, S.A. Hackney, N.J. Dudney, V. Thole, P.S. Phani, Nanoindentation of high purity vapor deposited lithium films: A mechanistic rationalization of diffusion-mediated flow. J. Mater. Res. 33, 1347-1360 (2018)

3. E.G. Herbert, S.A. Hackney, N.J. Dudney, V. Thole, P.S. Phani, Nanoindentation of high purity vapor deposited lithium films: A mechanistic rationalization of the transition from diffusion to dislocation-mediated flow. J. Mater. Res. 33, 1361-1368 (2018)

4. Y. Chung, C. Lee, Electrochemical behaviors of Indium. J. Electrochem. Sci. Technol. 3, 1-13 (2012)

5. M. Ashby: CES EduPack. Granta Design, (2019).

6. G. Feng, A. Ngan, Creep and strain burst in indium and aluminium during nanoindentation. Scripta Mater. 45, 971-976 (2001)

7. H. Li, A. Ngan, Size effects of nanoindentation creep. J. Mater. Res. 19, 513-522 (2004)
8. W. Li, R. Warren, A model for nano-indentation creep. Acta Metall. Mater. 41, 3065-3069 (1993)

9. B.Q. Han, T.G. Langdon, Factors contributing to creep strengthening in discontinuously-reinforced materials. Mater. Sci. Eng., A 322, 73-78 (2002)

10. G.M. Pharr, E.G. Herbert, Y.F. Gao, The indentation size effect: A critical examination of experimental observations and mechanistic interpretations. Ann. Rev. Mat. Res. 40, 271-292 (2010)

11 M. Rester, C. Motz, R. Pippan, Microstructural investigation of the volume beneath nanoindentations in copper. Acta Materialia 55, 6427-6435 (2007)

12. B.N. Lucas, An experimental investigation of creep and viscoelastic properties using depth-sensing indentation techniques, materials science and engineering, University of Tennessee, 1997

13. B. Lucas, W. Oliver, Indentation power-law creep of high-purity indium. Metall. and Mater. Trans. A. 30, 601-610 (1999)

14. R. Tilly, Defects in Solids (Wiley, Hoboken, New Jersey, USA, 2008).

15. R. Asaro, W. Tiller: Interface morphology development during stress corrosion cracking: Part I. Via surface diffusion. Metallurgical and Materials Transactions B, (1972)

16. W. Mullins: The Effect of Thermal Grooving on Grain Boundary Motion. Acta Met, 6, (1958).

17. C. Fincher, Y. Zhang, G. Pharr, M. Pharr, Elastic and plastic characteristics of sodium metal. ACS Appl. Energy Mater. 3, 1759-1767 (2020) 